Cuadernos de Lingüística Hispánica $n^{\circ} .24$

ISSN 0121-053X • ISSN en línea 2346-1829

Julio-Diciembre 2014, pp. 145-162

\title{
Influencia léxica del Muisca en el español actual clasificada en campos semánticos*
}

\author{
DIANA ANDREA GIRALDO GALLEGO** \\ Diana.Gallego@if.uib.no
}

Recepción: 13 de febrero de 2014

Aprobación: 21 de abril de 2014

Cómo citar este artículo: Giraldo Gallego, D. (2014). Influencia léxica del Muisca en el español actual clasificada en campos semánticos. Cuadernos de Lingüística Hispánica, 24, 145-162. Tunja: Uptc.

\footnotetext{
Artículo de investigación científica. Recoge resultados contenidos en la tesis de maestría En búsqueda de El Dorado. Préstamos de origen muisca en algunos municipios de Cundinamarca y Boyacá. Mis estudios han sido financiados por el programa Quota, gracias al convenio existente entre la Universidad de Bergen y la Universidad de Antioquia. Parte de la información contenida en este artículo la presenté como ponencia en el XXVII Congreso Nacional y I Internacional de Linguística, Literatura y Semiótica en la Universidad Pedagógica y Tecnológica de Colombia en el año 2012.

* Profesora ocasional en la Facultad de Comunicaciones de la Universidad de Antioquia (UdeA). Doctoranda en Español y Estudios Latinoamericanos por la Universidad de Bergen (UiB) donde hace su tesis sobre la presencia de elementos lingǘsticos del muisca en el español. Es magíster en Español y Estudios Latinoamericanos por la UiB y Traductora Inglés-Francés-Español por la UdeA. Es miembro del Grupo de Estudios Sociolingüísticos (GES) y del grupo de investigación sobre la lengua muisca Muysccubun.
} 
Influencia léxica del Muisca en el español actual clasificada en campos semánticos

\title{
Resumen
}

La presencia léxica del muisca en el dialecto cundiboyacense es una muestra de que esta influencia aún sigue vigente. El objetivo de este artículo es presentar los préstamos y posibles préstamos del muisca en el español actual clasificados en campos semánticos. Los datos se recolectaron en los municipios de Machetá y Tibirita en el departamento de Cundinamarca; Guateque, Sutatenza, Guayatá, La Capilla, Garagoa, Chinavita y Pachavita en el departamento de Boyacá. Los resultados se organizaron en siete campos semánticos: campo y cultivo; minería; espacio; animales; familia y ciclo de vida; cuerpo humano; recipientes, alimentación y vivienda. Se concluyó que los campos que presentan una mayor influencia son los que corresponden a cultivo y animales.

Palabras clave: préstamo léxico, español, muisca, campo semántico.

\section{Lexical influence of the Muisca language in current spanish, classified in semantic fields}

\begin{abstract}
The lexical presence of the Muisca language in Cundiboyacense dialect is a proof that its influence is still in force. The objective of this article is to present the loanwords and possible borrowings from Muisca in current Spanish, classified in semantic fields. The data were collected at the municipalities of Machetá and Tiribita in the department of Cundinamarca; Guateque, Sutatenza, Guayatá, La capilla, Garagoa, Chinavita y Pachativa in the department of Boyacá. The results were organized into seven semantic fields: country and farming; mining; space; animals; family and life cycle; human body; containers, food and housing. The study concluded that the most influential fields are those corresponding to farming and animals.
\end{abstract}

Key words: loanword, Spanish, Muisca, semantic fields. 


\title{
Influence lexicale du Muisca dans l'espagnol actuel, classée dans des champs sémantiques
}

\begin{abstract}
Résumé
La présence lexicale du Muisca dans le dialecte cundiboyacense est une preuve du fait que cette influence existe encore. L'objectif de cet article est celui de présenter les emprunts et les possibles emprunts du Muisca dans l'espagnol actuel, classés dans des champs sémantiques. Les données ont été collectées dans les communes de Machetá et Tibirita dans le département de Cundinamarca; Guateque, Sutatenza, Guayatá, La Capilla, Garagoa, Chinavita et Pachavita dans le département de Boyacá. Les résultats ont été organisés dans sept champs sémantiques: campagne et culture; minerie; espace; animaux; famille et cycle de vie; corps humain; récipients, alimentation et logement. On a conclu que, les champs ayant une influence plus importante sont ceux de la culture et des animaux.
\end{abstract}

Mots clés: emprunt lexical, espagnol, Muisca, champ sémantique.

\section{Influência léxica do Muisca no espanhol atual classificada em campos semânticos}

\section{Resumo}

A presença léxica do muisca no dialeto cundiboyacense é uma amostra de que esta influência ainda segue vigente. 0 objetivo deste artigo é apresentar os empréstimos e possíveis empréstimos do Muisca no espanhol atual classificados em campos semânticos. Os dados foram coletados nos municípios de Machetá e Tibirita no departamento de Cundinamarca; Guateque, Sutatenza, Guayatá, La Capilla, Garagoa, Chinavita e Pachavita no departamento de Boyacá. Os resultados se organizaram em sete campos semânticos: campo e cultivo; mineração; espaço; animais; família e ciclo de vida; corpo humano; recipientes, alimentação e moradia. Concluiu-se que os campos que apresentam uma maior influência são os que correspondem a cultivo e animais.

Palavras chave: empréstimo léxico, espanhol, Muisca, campo semântico. 


\section{Introducción}

La importancia de la lengua muisca para la historia de Colombia radica en que durante la época de la Colonia tuvo el estatus de lengua general; se habló en la que hoy es la capital del país y se abrieron cátedras para su enseñanza-aprendizaje. Dentro de los trabajos descriptivos del muisca que se hicieron en ese momento se encuentran las gramáticas y, por otro lado, las traducciones al muisca de los catecismos y oraciones católicas que dan cuenta del uso de la lengua en un contexto de evangelización. En la época contemporánea, los estudios sobre sus vestigios en el español de Colombia surgieron gracias a reconocidos hispanistas como don Ezequiel Uricoechea, don Rufino José Cuervo, don José Joaquín Montes Giraldo, entre otros, quienes dedicaron sus trabajos no solo a los estudios descriptivos del español, sino a los de la influencia de las lenguas indígenas, y en este caso particular del muisca, en el uso local.

Como en la época de la Conquista, la mayor influencia de las lenguas indígenas en el español se dio en lo relacionado con la flora y la fauna (Montes Giraldo, 1986, p. 335; Enguita Utrilla, 2004, p. 22) dada la diversidad y novedad que representaron para los conquistadores, muchos de los elementos léxicos que sobreviven en la actualidad están relacionados con plantas y animales. Esto es lo que mostraremos a partir de los datos que recogimos en algunos municipios ubicados en el antiguo territorio muisca.

Los datos que aquí presentamos los agrupamos en campos semánticos, es decir, en grupos integrados por unidades léxicas relacionadas semánticamente en un campo determinado. Un campo semántico lo entendemos aquí como «un grupo de palabras íntimamente relacionadas por su significado que, frecuentemente, quedan subsumidas bajo un término general» (Paulín Pérez, 2006, p. 211). Los términos generales que nosotros proponemos en este artículo siguen la misma división que empleamos en el cuestionario que utilizamos para la recolección de los datos: campo y cultivo; minería; espacio; animales; familia y ciclo de vida; cuerpo humano; recipientes, alimentación y vivienda (Giraldo Gallego, 2011). Rodríguez (2005, pp. 109-110) propone la siguiente clasificación de campos semánticos según la cantidad o índole de las unidades léxicas que podrían integrar cada 
campo semántico: cerrado (meses del año, días de la semana), abierto (especies de animales o plantas), graduales (antónimos) y asociativos (como los diccionarios ideológicos). Consideramos, entonces, que los datos que aquí mostraremos se clasifican dentro de los campos semánticos abiertos, pues si bien es cierto que tenemos un número determinado de préstamos o posibles préstamos de origen muisca (141), en un futuro esta cantidad podría variar en la medida en que se podrían identificar nuevos préstamos. Esperamos que estos resultados sean de gran utilidad para la comunidad académica y para la sociedad en general, ya que representan el patrimonio vivo de una lengua lamentablemente extinta.

\section{Antecedentes}

A finales del siglo XIX empezaron a surgir estudios que refieren la influencia léxica del muisca en el español hablado en Cundinamarca y Boyacá. Entre estos, podemos mencionar los estudios de Ezequiel Uricoechea, quien en 1871, con su obra Gramática, vocabulario, catecismo i confesionario de la lengua chibcha. Según antiguos manuscritos anónimos e inéditos, aumentados i correjidos, presentó nueve voces chibchas que se conservaban en el lenguaje de Bogotá con sus respectivas etimologías: chajuá < <hahuana , chiguacá < <chiguacás, chiza < <isa〉, chúcua < <chupqua>, cuba < <uhuba〉, futearse < <afutynsuca , guapucha < «gua pquyhyza , iomogó < <iomgo> y tunjo < <chunso〉.

Rufino José Cuervo a partir de la $1^{\text {a }}$ edición de sus Apuntaciones críticas sobre el lenguaje bogotano (aquí tomaremos en cuenta la $5^{\text {a }}$ edición), confrontó algunas voces escuchadas en Bogotá con el manuscrito Vocabulario de la Lengua Mosca ó Chibcha localizado en la Biblioteca Nacional de Colombia y agrega: "en él no hemos encontrado de las voces indígenas sobrevivientes á la conquista sino las que á continuación copiamos" (pp. 642-643): cuan < <pquane〉, cuba < <uhuba〉, chajuá/chajuán < <suaz/ chahanabcuscua, chisa < <isa , chisgua < <hisua, chitearse $<$ <azitynsuca, chucua $<$ <chubcua>, chucho < chuza/achuhuzansuca , chusque < chusquy, futearse < <afutynsuca , guapucha < <guapquyhyza, guascas < <quysca/huazyca $>$, quincha < <quynza, sote < <sote, totear/totazo < btohotynsuca y yomogó < <iomza/iemuy/iomgy». También presentó otras voces: cubias, mojas/mojanes, víchiro, bijuacá, chisacá; los terminados en -uba, -ubo: curuba, uchuba, cucubo que coincidirían con «uba> 'flor', 'grano'; y otros probables chichagúy [sic] y sal vijua.

Fernando Antonio Martínez en su artículo de 1977 A propósito de una gramática chibcha y a propósito de algunas supervivencias chibchas del habla de Bogotá, además de incluir las voces expuestas por Cuervo ${ }^{1}$, presentó otras con cierta probabilidad. Sin

\footnotetext{
Se basa en la $1^{\text {a }}$ edición de Cuervo (1867-1872).
} 
embargo, el artículo quedó inconcluso ${ }^{2}$ y algunas de esas voces quedaron por confirmar. Por ejemplo, el autor se pregunta "no sobrevivirá ch. guara 'mozo'?" (ubi supra: p. 25). A continuación, exponemos aquellas supervivencias que se muestran definidas en este trabajo: chircate < <chin-cate ${ }^{3}$, uyuba y cuchuba < <quye uba, cuchubo < <cujupcua $>$ yiquitoque.

En 1978, José Joaquín Montes Giraldo, en su artículo Fitónimos de sustrato en el español del altiplano cundiboyacense y dialectos muiscas, expuso algunos fitónimos muiscas y presentó un importante hallazgo: la presencia de un rasgo dialectal muisca o chibcha ante la alternancia $c h-r r$ en nombres de plantas. Este fenómeno fue localizado en el altiplano cundiboyacense gracias a las encuestas del Atlas lingüístico-etnográfico de Colombia (ALEC). El autor también propuso una serie de palabras de cierto o probable origen muisca de acuerdo con dos criterios:

1. La cantidad de fitónimos recolectados que terminan en $c a$, que, cha y che, y que las ubicó como un probable sufijo clasificador para nombres de plantas: ajondoque, (al) payaca, chaque-raque, chipaca, chipaque, chisacá-risacá, choque-cuque-soque, chuguacá, churca, chusca, chusque, cuarque, guaque, janche, quiche, rubacá, rúchica, susca - chusca, tequito, uche, unca.

2. La relación del fitónimo con topónimos o con elementos muiscas: aguaquín, chío-chibo, chisgua, tabio.

También presentó una lista de fitónimos de una menor probabilidad de ser de origen muisca: buncón, charne, chobo, guaba, gurrubo, ipa, cu(o)cubo, tobo, tótomo, tutúes, ира у иро. Finalmente, estableció la distribución geográfica de la alternancia $c h$ - rr que pareció concordar con las diferencias "más notorias que se daban entre la lengua de la parcialidad del Zipa de Bogotá -la dominante en la época de la llegada de los españolesy la de los dominios del Zaque de Tunja" (Montes Giraldo, 1978, p. 48). Incluyó además otras alternancias: chacua - racua o roca, chiso - riso, socha o sote - roche, y chugua ruba.

En $1984^{4}$, María Luisa Rodríguez de Montes presentó 45 muisquismos léxicos que aparecían en los seis tomos del ALEC y otra lista de probables muisquismos. Así mismo, mostró derivaciones y variaciones morfo-fonológicas de algunos de ellos y la localización diatópica del registro. Entre los criterios que tuvo para la selección de estas voces resaltamos:

En el artículo hay un pie de página del Instituto Caro y Cuervo donde lamenta el fallecimiento del autor, razón por la que este no se terminó.

El autor toma las etimologías del vocabulario de Uricoechea (1871); sin embargo, la palabra muisca que aparece en este vocabulario es <chircate> (p. 194)

Arango (1995, pp. 124-132) expone los muisquismos presentados en esta publicación.

\section{0}


"por encontrarse las unas en el vocabulario de la lengua muisca editado por Ezequiel Uricoechea, o por haber demostrado que son muiscas tanto el mismo Uricoechea como Rufino José Cuervo" (Rodríguez de Montes, 1984, p. 43). Otros criterios incluyen la presencia de la variante dialectal $c h-r r o$ las terminaciones -ca, -que, - cha, -che $\mathbf{s}^{5}$ abagó < a abago bquyscua>, acua < <abquye>, alpayaca <-ca>, amero (por el lexema am $(u)$ ), cuán < <pcuamne>, cocubo/cucubo/ubo/chubas <-uba/-ubo>, cuba < <cuhuba〉, cubios < <cubia/ cubios, cuchuco/cochuco < <quychyquy/aquychyquy>, cute < <cute/zapquy cutys, chagual racualcharua $($ ch rr), chajuá < <chahuana>, changua/chingua/chirgua (por los participios del verbo <zeguascua: <chaguasca/chaguaca/chaguanga , chichaguy, chingue/ chinga < <hine/chinz>, chisa/sisa < <isa〉, chisacá $($ ch $\sim$ rr $)$, chisgua/risgua/sarcua, chocá/choca/chocó/choco < zzocaz/zoque〉, chonque <-que〉, chucua/chuquio/chuquial/ chupia/chuquia/chucal < <chupqua〉, chucha/chucho < <aba chuza/achuzansuca/ achuhuzansuca , chugualruba $(c h \sim r r)$, chuguacálrubacá $(c h \sim r r)$, chunguy < <chumne + gye>, chusque < <chusque>, ficaljicaljiquia < fica>, fuchalfuche/jucha < fazua/suize/ iza , gatica < 〈gata>, guan < <guan>, guapuchalaguapucha < <gua pquyhiza, guata/guate < 〈guat zona/guatin zona/guat azone>, guayome <iomza/iomuy, ibajuquiar/ibajuca < 〈guasgua zebquiguasuca〉, moján/mohán/muán (por la mención de los cronistas a este ser mitológico), oche/uche <-che>, quincha < <quynza , sote/socha/soche/roche < <sote/sohoza , suco/suca < <suhuca , suque < <suque, tunjo < <chunso/chunsua/chunsua guia , uchuba $<$-uba/-ubo〉, utear < <abutynsuca , víchiro, yomogó/yemogó < <aiomgo bquyscua〉.

En 1992, José Rozo Gauta presentó 13 muisquismos tomados de algunas coplas de Cundinamarca y Boyacá. Expuso la unidad léxica en contexto, su explicación cultural y, en algunos casos, histórica y etimológica, ya que como él mismo dijo: "el aspecto lingüístico nos sirve de pretexto para describir contenidos y sentidos históricos, etnográficos y de otros órdenes" (p. 56): abago, amero, arremuesco, babaguy, cacacuy/quicacuy, cusca/cosca, chanchirienta/chanchirosa, chanchiros, changua, chimbilá, chingada, chingue, juaica ${ }^{6}$.

En 2006, María Stella González de Pérez presentó ocho posibles muisquismos tomados del Glosario lexicográfico del Atlas lingüístico-etnográfico de Colombia (ALEC) (Montes Giraldo, et al., 1986). Propuso las etimologías tomadas de González de Pérez (1987), Quesada Pacheco (1991) y la transcripción fonética con base en su trabajo Aproximación al sistema fonético-fonológico de la lengua muisca publicado en 2006: bachiquiar/ basicar < 〈bxisqua〉, chichacuar < <bchichysuca〉, chichagüi/chichaguy $<$ hybaz

\footnotetext{
En el transcurso del artículo también se confirman otras voces por la terminación - $u b a,-u b o$, y por el registro de la palabra en el antiguo territorio de asentamiento muisca.

Parece ser que no es un muisquismo léxico, pues de acuerdo con las explicaciones del autor, se deduce que es el significado el que sobrevive y no el significante: «dicho personaje sería Bochica o un sacerdote del culto solar» (Rozo Gauta, 1992, p. 69).
} 
ichichanyquy/hybaz ichicha asaquy, guasoquiar < <higuasuca , guayomas/guayumbas < <neiomy/niomy», pacha < <pabcha〉, suque < <achisuquezona〉 y unche < <uze〉.

De todas estas publicaciones sobre la influencia léxica del muisca en el español, concluimos que las voces muiscas registradas por Uricoechea (1871) y Cuervo (1907) pertenecen a aquellas escuchadas en Bogotá, pero no a las de lugares distantes de esta capital y que coinciden con el antiguo territorio de asentamiento muisca. Las coplas, como manifestación cultural, también representan un importante objeto de análisis, ya que pueden incluir unidades léxicas de origen muisca como lo presentó Rozo Gauta (1992). Aunque el objetivo del $A L E C$ no fue recolectar léxico de origen indígena, sino registrar el habla popular del territorio colombiano, ha sido de gran utilidad para poner en evidencia unidades léxicas de origen muisca registradas durante la implementación de las encuestas, además de dejar al descubierto la presencia de un rasgo dialectal que podría corresponder con los dominios del Zipa, por un lado, y los del Zaque, por el otro. Así pues, esta introducción y el léxico de origen muisca que mostraremos en este artículo nos brindan un panorama sobre aquellos campos semánticos que dejaron su huella en el español de Colombia.

\section{Metodología}

La clasificación que aquí presentamos la tomamos del cuestionario que utilizamos para la recolección de los datos en el trabajo de maestría de Giraldo Gallego (2011). Estos datos los recolectamos en el año 2010 en los municipios de Machetá y Tibirita en el departamento de Cundinamarca; Guateque, Sutatenza, Guayatá, La Capilla, Garagoa, Chinavita y Pachavita en el departamento de Boyacá (ver Mapa 1). Entrevistamos a personas oriundas de la zona y cuyos padres y pareja provenían del mismo lugar, mayores de 50 años, habitantes del área rural y con ninguna o poca instrucción escolar. Para las entrevistas implementamos un cuestionario con 852 preguntas divididas en siete campos semánticos: campo y cultivo; minería; espacio; animales; familia y ciclo de vida; cuerpo humano; recipientes, alimentación

y vivienda. Para la creación de este instrumento seguimos a Flórez, et al. (1981-1983) y a Quesada Pacheco (1992). 


\section{Mapa $1^{7}$}

Municipios en donde se implementó el cuestionario Machetá, Tibirita, Guateque, Sutatenza, Guayatá, La Capilla, Garagoa, Chinavita, Pachavita

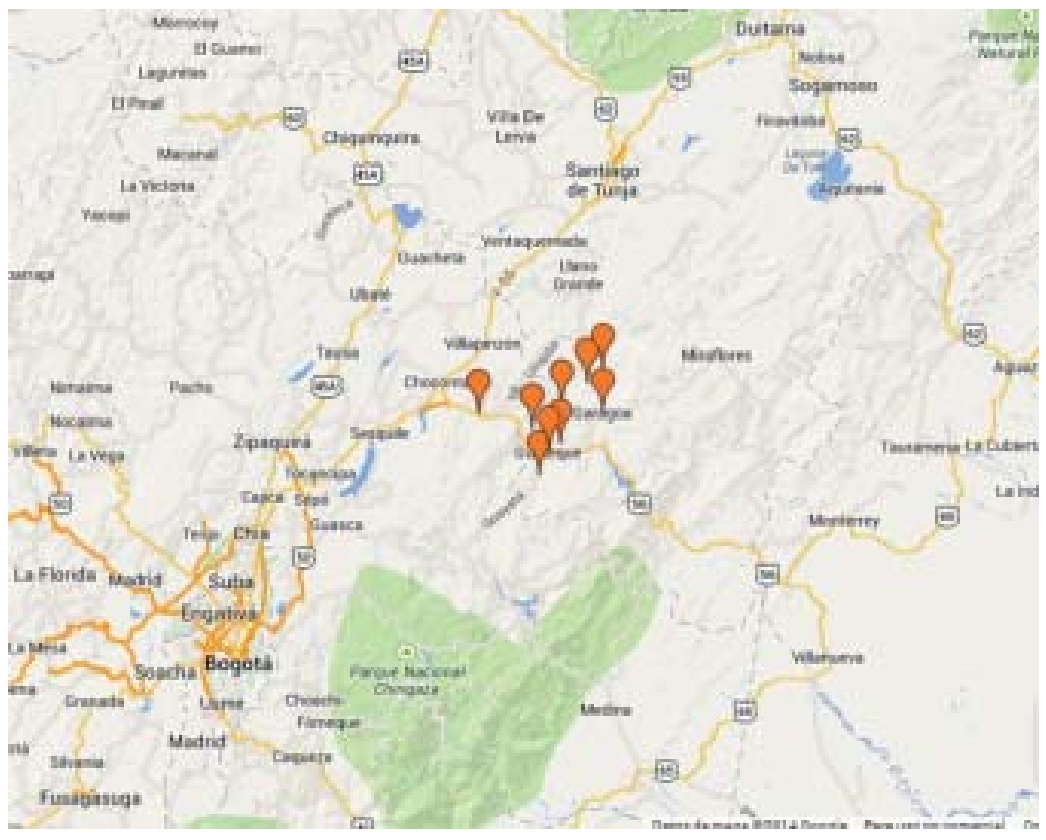

Inicialmente, obtuvimos 1.733 unidades léxicas. Posteriormente, descartamos aquellas de origen hispano o de otros orígenes y aquellas que se escucharan en otros países como, por ejemplo, México, Perú, Argentina o Chile, ya que la unidad léxica por analizar podía tener un origen en las respectivas lenguas locales, más que en el muisca. Para la selección y organización del material recopilado, empleamos los siguientes criterios: referencial, etimológico-semántico, genético, geográfico, fonético, histórico y dialectal ${ }^{8}$. A partir de estos criterios establecimos 141 unidades léxicas como préstamos de origen muisca y de posible origen muisca. Como mencionamos más arriba, estos resultados los presentamos en este artículo distribuidos en siete campos semánticos. Decidimos incluir la definición de cada uno de los lemas dado que temíamos que algunos de estos no fueran familiares para el lector. La clasificación diatópica de los registros y los criterios empleados para su clasificación como de origen muisca o de posible origen muisca se pueden consultar en Giraldo Gallego (en prensa). Las definiciones de cada uno de los lemas los construimos a

\footnotetext{
Este mapa fue realizado en Google Maps: https://maps.google.es

Una descripción más detallada puede verse en Giraldo Gallego (en prensa).
} 
partir de las respuestas brindadas por los informantes. Los nombres científicos de plantas y animales los tomamos del Nuevo diccionario de colombianismos (1993); del Diccionario de americanismos (2010), de Montes Giraldo (1981) y de la Organización para la Educación y Protección ambiental (OPEPA). En algunos de los préstamos y posibles préstamos se presentaron ciertas variantes fonéticas que incluimos aquí en una misma entrada y separadas por una barra inclinada (/).

\section{Lista de préstamos y posibles préstamos de origen muisca agrupados por campos semánticos}

\subsection{Campo y cultivo}

Abagó: 1. 'La papa, arracacha, batata o yuca más grande'. 2. 'Lo mejor de la cosecha de maíz'. 3. Metafóricamente, 'niño pequeño y gordo'.

abaquín: 'árbol de la zona'.

achisua: 'planta de la que se extrae harina para uso comestible' (Canna edulis).

amero: 'hoja que envuelve la mazorca'.

balú/baluy: 'árbol de tallo espinoso y flores de color rojo' (Erythrina edulis).

bayago: 'cierta clase de árbol frutal'.

bijitoque/fijitoque: 'cada una de las partes en que se divide la hoja del fique'.

chacua: 'cada una de las partes en que se divide la hoja del fique'. 2. 'Una porción de fibras de fique'.

chacuar: 'tomar una porción de fibras de fique y torcerlas'.

cheraco: 'cierto árbol de la zona'.

chicalá: 'nombre que incluye varias especies de árboles que se caracterizan por sus flores vistosas' (Tabebuia spp.).

chigua/chisua/rigua: 'hierba cuyo fruto es de color negro brillante, similares a los del café' (Canna indica).

chilada: 'medida equivalente a un chile o costal'.

chile: 'costal tejido en fique'.

chin: 'caña que se emplea como material para la elaboración de canastos, techos y otros usos' (Arundo donax).

chipaca: 'hierba de la que nacen unas flores amarillas y blancas'. También se utiliza como planta medicinal (Bidens pilosa).

chisa: 'fruto del chiso'.

\section{4}


chisaca/chisacá/risacá: 'hierba que se emplea para usos medicinales' (Spilanthes americana).

chiso: 'árbol maderable, da un fruto redondeado y rojo' (Myrica sp.).

chocho: 'árbol de semillas de color rojo brillante que se emplean para la elaboración de artesanías' (Erythrina rubrinervia).

chuba/chugua/ruba: 1 . 'hierba de hojas acorazonadas y comestibles que produce un tubérculo comestible' (Ullucus tuberosus). 2. 'Tubérculo comestible'.

chulque/chusque: 'caña de los páramos que se emplea para hacer canastos y techos' (Chusquea spp.).

chuque: 'árbol del que nacen flores blancas en época de floración' (Viburnum triphyllum).

chuscal: 'lugar donde crece la caña chusque'.

coca: 'hoja que envuelve la mazorca'.

cuan: 'cada una de las partes en que se divide la hoja del fique'.

cuarque/guarque: 'árbol del que cuelga cierto musgo' (Tillandsia usneoides).

cubio: 'tubérculo comestible de color morado' (Tropaeolum tuberosum).

cura/curapo: 'aguacate'.

curapa: 'Semilla del aguacate'.

curo: 'árbol del aguacate' (Persea americana).

curuba/guruba: 'fruto del curubo'.

curubo: 'planta enredadera cuyo fruto es alargado y de color amarillo' (Passiflora antioquensis).

cute: 'cáscara de la papa o la yuca'.

desamerar: 'quitar la hoja que cubre la mazorca'.

desenguichar: 'quitar el guiche'.

enchinar: 'unir o amarrar cañas de chin que sirven como techo'.

enchuscado: 'techo construido con caña de chin o chusque'.

enchuyada: 'papa de no muy buen sabor'.

escachar: 'cortar la hoja de la caña de maíz'.

escocar: 'quitar la hoja que cubre la mazorca'. 
Influencia léxica del Muisca en el español actual clasificada en campos semánticos

esjiquiar: 'cortar la hoja de la caña de maíz o de azúcar'.

fica/jica/jiquia/fiquia: 'hoja de la caña de maíz' .

fique/jique: 'planta de pencas de donde se extrae la cabuya' (Agave spp. y Furcraea spp.).

fiquera: 'terreno plantado de fique'.

fucha/jucha: 1. 'líquido que sale del fique'. 2. 'Prurito producido por el líquido que sale del fique'.

fuchoso/juchoso: 'objeto o planta que produce rasquiña cuando hace contacto con la piel'.

fute/jute: 'fruto descompuesto'.

futearse: 'descomponerse una fruta'.

gaque/guaque: 'árbol cuyas hojas son gruesas y redondeadas' (Clusia spp.).

guaba: 'hierba de la que crece una flor alargada con frutos pequeños' (Phytolacca icosandra).

guaca: 'hierba con flores de color amarillo. Es comestible' (Spilanthes americana).

guaiome/guayome: 'cosecha secundaria de papa, fríjol y/o arveja'.

guaney: 'cierta clase de árbol' (Tecoma spectabilis o Erythrina Poepigiana).

guasca: 'hierba de hojas con flores pequeñas de color blanco y amarillo en el centro' (Galinsoga urticaefolia).

guasguín: 'cierta clase de árbol. Es muy conocido por sus propiedades curativas' (Microchete conymbosa).

guiche/quiche: 'planta epifita que nace sobre las ramas de los árboles' (Tillandsia spp.).

gulupa/gurupa: 'planta enredadera y trepadora, su fruto es de color verde redondo' (Passiflora ornata).

jiyome: 'papa pequeña'.

jupa: 'clase de pasto'.

nuche: 'cierta clase de árbol'.

ocobo: 'árbol de flores amarillas, rosadas o blancas. En época de floración pierde todas sus hojas' (Tabebuia spp.).

oguín: 'árbol de la zona'.

\section{6}


pacha: 'dos frutos unidos'.

rebancá: 'clase de hierba que se usa como condimento para las sopas' (Brassica campestris).

riche: 'papa muy pequeña'.

ruya: 'bolas hechas de masa de maíz que se le echan a la sopa'.

situta: 'planta enredadera'.

sona: 'caña que se emplea como material para hacer canastos'.

sututa: 'cierta clase de pasto'.

tauquín: 'cierta clase de árbol de la zona'.

tibame: 'tipo de maíz amarillo'.

tibaquín: 'cierta clase de árbol'.

tíbar: 'arbusto de flores pequeñas, blancas y rosadas' (Escallonia paniculata).

tocua: 'cierta clase de árbol parecido al café'.

totazo: 'golpe'.

tote: 1. 'mazorca con algunos granos'. 2. 'Hierba de hojas largas' (Dichromena ciliatum).

totear: 'quebrar o reventar una cosa'.

totuda, ta: 'mazorca con algunos granos'.

tótomo: 'cierta clase de hierba'.

toy: 'semilla de la papa que crece después de recogida la cosecha'.

toyal: 'campo lleno de maleza'.

toyo: 1 . 'Recipiente hecho de calabazo'. 2. Metafóricamente, 'orejas'.

uche: 'árbol de buena madera'.

uchuva: 'planta con flores colgantes. Su fruto es de color amarillo' (Physalis peruviana).

unche: 1.'Bagazo o líquido que sale del fique'. 2. 'Residuo del maíz'.

\subsection{Espacio}

Chichaguyal: 'depósito natural de agua'.

chitearse: 'quebrarse'.

chucua/chucual: 'terreno pantanoso o húmedo donde nacen juncos'. 
Influencia léxica del Muisca en el español actual clasificada en campos semánticos

\subsection{Animales}

Bababuy/babaguy: 'Ave de plumaje negro, pecho amarillo y manchas blancas en sus alas' (Pheucticus aureoventris, Fam. Fringillidae) .

caica: 'ave acuática de pico largo y plumaje café' (Gallinago nobilis y Gallinago sp.).

chafí/chagí/chafiro/chagiro: 'ave de plumaje amarillo con negro' (Icterus chrysater).

chanilla: 'huevos que dejan las moscas en ciertas materias orgánicas'.

chía/chisa/chila: 'larva que se da en el cultivo de papa' (Melolonthidae; Phyllophaga spp).

chinguy/chunguy: 'cierta clase de cucarrón'.

chisga: 'cierta clase de pájaro' (Sporophila sp. y Tiaris sp., Fam. Fringillidae).

chucha/runcho: 'mamífero marsupial de pelaje grisáceo o negruzco' (Didelphis marsupialis).

chulo, la: 'Buitre de plumaje negro y cabeza de color grisáceo' (Coragyps atratus, Fam. Cathartidae).

chunguy/chinguy: 'cierta clase de cucarrón'.

fara/jara: 'mamífero marsupial de pelaje grisáceo o negruzco' (Didelphis marsupialis).

guala/guara: 'Ave rapaz de plumaje negro, cabeza sin plumas y de color rojo' (Cathartidae, Cathartes aura).

quete: 'gusano que afecta el cogollo del maíz, también se le llama trozador'.

quetear: 'cortar'.

quincha: 'pájaro colibrí'.

soche: 'venado mediano de color rojizo'. (Mazama spp., especialmente Mazamma ruffina en los Andes).

sote: 'pulga que se mete entre las uñas y deposita sus crías allí. Estas producen un fuerte picazón' (Rynchoprion penetrans).

tocha: 'cierta clase de serpiente'.

toche: 'ave con plumaje negro y pecho amarillo' (Icterus chrysater, Fam. Icteridae).

yátaro: 'ave de pico largo y ancho, de plumas de color verde o combinadas con amarillo, rojo o negro' (Fam. Ramphastidae).

158 
ziota: 'cierta clase de pájaro' (Turdus fuscater).

\subsection{Familia y ciclo de vida}

Cuba: 'hijo menor'.

cocacuy/cucacuy/cucuyé/quicacuy:'ser mítico que cuida los tesoros que dejaron los indígenas. Con un orificio en la uña crecida de su dedo pulgar tiene un orificio por donde silba'.

guate: 1. 'caña de la zona'. 2. 'Persona que vive en la montaña y no conoce el llano'.

mohán/moján: 'Ser mítico que cuida de tesoros. Sus historias están relacionadas con el agua'.

tunjo: 'ser mitológico'.

\subsection{Cuerpo humano}

Chingao: 'persona mal vestida'.

chirao: 1. 'prenda de vestir en mal estado'. 2. 'Prenda de vestir fabricada con material ordinario'.

chiro: 1 . 'pedazo de trapo que se usa para limpiar. Ropa vieja o que no está en muy buen estado'.

chirosa: 'gallina de plumas encrespadas'.

chirudo, da: 'tejido que no queda bien hecho'.

choqui: 'cabeza'.

chuchuba/turupa: 'pelota que se forma cuando uno se da un golpe en la cabeza'.

chuto: 'cabello muy rizado'.

enchirada: 'vestido con ropa vieja o desgastada'.

\subsection{Recipientes, alimentación y vivienda}

Achuyarse: 'Endurecerse el alimento después de cocido, especialmente, la yuca'. 2. 'Quedar el alimento de mal sabor'.

boroi/broi: 'guarapo, bebida fermentada'.

changua: 'caldo que se toma al desayuno preparado con agua, pan de maíz, papa y leche'.

chigua: 'bolso tejido en fique'.

china: 'instrumento para avivar el fuego. Generalmente, su tejido es de paja'. 
Influencia léxica del Muisca en el español actual clasificada en campos semánticos

chipa: 'alambre o cabuya enrollada'.

chiqui: 1. 'Rodete sostenido del techo y sobre el que se ponen recipientes con alimentos para resguardarlos de los animales'. 2 . 'Rodete que se pone en la cabeza para transportar las ollas de barro'.

choco: 'recipiente hecho de calabazo'.

chuyo, ya/ruya'rucha: 1 . 'yuca o alimento que no se ablanda al cocinarlo'. 2. 'Papa vieja'.

3. 'Palo que se quiebra con facilidad'.

coica: 'caparazón del armadillo'.

cona: 'caparazón del armadillo'.

corroica/corrosca: 'caparazón del armadillo'.

cuchuco: 'sopa tradicional hecha a base de maíz y harina. Este último ingrediente se le agrega para que cobre espesor'.

cutada: 'bebida fermentada, normalmente, guarapo'.

enchipar: 'enrollarse un animal o enrollar un material como un lazo o alambre'.

guchubo: 'canasto con manija'.

quisba/quisbón: 'arepa de maíz tierno'.

\section{Conclusión}

Los préstamos de origen muisca y de posible origen muisca que recolectamos en el año 2010, los presentamos en este artículo clasificados en campos semánticos. La cantidad de lemas de origen muisca o posible origen muisca figuran en cada campo semántico así: campo y cultivo (86), espacio (3), animales (21), familia y ciclo de vida (5), cuerpo humano (9), recipientes, alimentación y vivienda (17). El campo semántico de la minería no se incluyó en este listado, debido a que en los datos no identificamos alguna unidad léxica que pudiera clasificarse como de origen muisca o de posible origen muisca; sin embargo, mencionamos dicho campo debido a que estuvo incluido en el cuestionario que se implementó para la recolección de los datos. Aquellos campos semánticos que presentan mayores registros corresponden con el de campo y cultivo y el de animales; le siguen el de recipientes, alimentación y vivienda, cuerpo humano y familia y ciclo de vida. Por último, estos resultados nos permiten dar cuenta del campo semántico que se ha mantenido más influenciado, y afirmar que la influencia muisca en el español aún sigue vigente. 
Diana A. Giraldo Gallego

\section{Referencias bibliográficas}

Anónimo. (Sin data). Diccionario y gramática chibcha. Manuscrito 158. Bogotá: Biblioteca Nacional de Colombia (Sin publicar).

Anónimo. (Sin data). Gramática, confesonario y vocabulario en lengua mosca. Manuscrito II/2922. Madrid: Real Biblioteca del Palacio Real (Sin publicar).

Anónimo. (Sin data). Vocabulario mosco de 1612. Manuscrito II/2923. Madrid: Real Biblioteca del Palacio Real (Sin publicar).

Anónimo. (Sin data). Vocabulario mosco de 1612. Manuscrito II/2924. Madrid: Real Biblioteca del Palacio Real (Sin publicar).

Arango, M. A. (1955). Aporte léxico de las lenguas indígenas al español de América. Barcelona: Puvill Libros S. A.

Constenla Umaña, A. (1984). Los fonemas del muisca. En: Estudios de lingüística chibcha. Tomo III, pp. 65-111.

Constenla Umaña, A. (1988). Indicios para la reconstrucción de clasificadores en el sintagma nominal protochibcha (1). En: Revista de filología y lingüistica de la Universidad de Costa Rica, XIV (2), pp. 111-118.

Cuervo, R. J. (1907). Apuntaciones críticas sobre el lenguaje bogotano. Con frecuente referencia al de los países de Hispano-América (V ed.). París: A. \& R, Roger y $\mathrm{F}$. Chernoviz, Editores.

Diccionario de americanismos / Asociación de Academias de la Lengua Española. (2010). Perú: Santillana.

Enguita Utrilla, J. M. (2004). Para la historia de los americanismos léxicos. Frankfurt am Main: Peter Lang.

Flórez, L. (1981-1983). Atlas lingüístico-etnográfico de Colombia (ALEC). Tomo I, II, III, IV, VI. Bogotá: Instituto Caro y Cuervo.

Giraldo Gallego. D. A. (2011). En búsqueda de El Dorado. Préstamos de origen muisca en algunos municipios de Cundinamarca y Boyacá. Universidad de Bergen (Tesis de maestría).

Giraldo Gallego, D. A. (En prensa). Préstamos de origen muisca en Cundinamarca y Boyacá. En: Estudios de Lingüística Chibcha.

González de Pérez, M. S. (1987). "Diccionario y gramática chibcha". Manuscrito anónimo de la Biblioteca Nacional de Colombia. Transcripción y estudio histórico-analítico. Bogotá: Instituto Caro y Cuervo.

González de Pérez, M. S. (2006a). Probables muisquismos, probables étimos. Recuperado de http://especiales.universia.net.co/vigia-del-idioma/numero-10-marzo-2006/probablesmuisquismos-probables-etimos.html 
Influencia léxica del Muisca en el español actual clasificada en campos semánticos

González de Pérez, M. S. (2006b). Aproximación al sistema fonético-fonológico de la lengua muisca. Bogotá: Instituto Caro y Cuervo.

Martínez, F. A. (1977). A propósito de una gramática chibcha y a propósito de algunas supervivencias chibchas del habla de Bogotá. En: Thesaurus: Boletín del Instituto Caro y Cuervo. Tomo 32 (1), pp. 1-23.

Montes Giraldo, J. J. (1978). Fitónimos de sustrato en el español del altiplano cundiboyacense y dialectos muiscas. En: Thesaurus, Boletín del Instituto Caro y Cuervo. Tomo XXXIII (Vol. 1), pp. 41-54.

Montes Giraldo, J. J. (1981). Medicina popular en Colombia. Vegetales y otras sustancias usadas como remedios. Bogotá: Instituto Caro y Cuervo.

Montes Giraldo, J. J., et al. (1986). Glosario lexicográfico del Atlas lingüístico-etnográfico de Colombia (ALEC). Bogotá: Imprenta patriótica del Instituto Caro y Cuervo.

Montes Giraldo, J. J. (1986). El influjo indígena en el español de Colombia. Caracterización sumaria. En: Revista de Filología Románica. (IV), pp. 335-340.

Nuevo diccionario de colombianismos (1993). (Tomo I). Santafé de Bogotá: Imprenta patriótica del Instituto Caro y Cuervo.

Organización para la Educación y Protección Ambiental (OPEPA). Recuperado de http:// www.opepa.org/

Paulín, Pérez, G. (2006). Rudimentos del lenguaje articulado (apuntes para el estudiante de Comunicación). México. Universidad Nacional Autónoma de México.

Quesada Pacheco, M. Á. (1991). El Vocabulario mosco de 1612. En: Estudios de lingüística chibcha. Tomo X, pp. 29-99.

Quesada Pacheco, M. Á. (1992). Atlas lingüístico-etnográfico de Costa Rica, cuestionario. San José: Editorial Tecnológica de Costa Rica.

Rodríguez, J. P. (2005). Gramática gráfica al juampedrino modo. Barcelona: Ediciones Carena.

Rodríguez de Montes, M. L. (1984). Muisquismos léxicos en el Atlas lingüístico-etnográfico de Colombia (ALEC). Homenaje a Luis Flórez. En: Publicaciones del Instituto Caro y Cuervo. (LXIX) pp. 1-55.

Rozo Gauta, J. (1992). Muisquismos en las coplas cundiboyacenses. En: J. Morales, \& E. Villa (Ed.), El folclor en la construcción de las américas. VI Congreso de Antropología en Colombia pp. 53-73. Santafé de Bogotá: Universidad de los Andes.

Uricoechea, E. (1871). Gramática, vocabulario, catecismo i confesionario de la lengua chibcha. Según antiguos manuscritos anónimos e inéditos, aumentados $i$ correjidos. París: Maisonneuve i Cia., Libreros editores.

162 